\title{
Based on Internet + VSDM and WebGIS Application and Analysis of Corn Fertilizing Decision-Making System
}

\author{
Yuxia Yao, ${ }^{1, a}$, Guowei Wang ${ }^{2, b}$, Qingru Sui ${ }^{1, c}$ \\ ${ }^{1}$ The Information Engineering College of Changchun University of Science and Technology, Ji Lin Chang \\ Chun 130600 China \\ ${ }^{2}$ The Information Technology College of Jilin Agricultural University, Ji Lin Chang Chun 130118 China \\ aemail:yx--yao@163.com; 41422306@qq.com;'email: 404409709@qq.com
}

Keywords: WebGIS, Internet +, corn, intelligent decision making system

\begin{abstract}
In recent years, with the rise of the Internet + and geographic information system technology, the traditional GIS has a new development space, let a lot of further application of spatial data for agricultural decision making analysis on the space provided technical support. In Internet + corn fertilizing decision-making in the process of application system, and accumulated a large number of agricultural spatial data, to organize and manage these data, better get the corresponding knowledge and management, analysis and decision-making, has developed "WebGIS corn intelligent decision-making system based on Internet +" for application. Using ArcIMS system realizes the network geographic information system basic operation, new mode based on tiles splicing ways of network map browsing, when pan, in IE will have the effect of image stitching, shorten the waiting time for the user psychology, also avoid a lot of pictures and large amount of data transmission, using the method of analytic hierarchy process (ahp) and fuzzy equivalence matrix, realized the space weighted fuzzy clustering algorithm, using F distribution, get the optimal classification number.
\end{abstract}

\section{Introduction}

Due to the spatial differences between corn fields is based on soil, soil nutrient spatial variation of the determination of precise fertilization, in order to ensure that farmers adjust measures to local conditions of fertilization, meet the need of national industrial structure adjustment of agriculture environment. It is the most effective way to achieve efficient, orderly and centralized management of the data of different space scales, data of different time periods and different formats in uniform coordinate system. The application of digital technology to establish a new mode of modern agriculture management, the transformation of traditional agriculture to modern agriculture, and promote the development of circular agriculture have very important practical significance ${ }^{[1]}$. Based on this idea, developed a fuzzy Data Mining based on the Visual space of the Internet + (Visual Spatial Data Mining, VSDM) and network geographic information system (WebGIS) of corn intelligent decision-making system is very necessary, the system could be used for agricultural production and management of various departments to provide decision-making reference information, provide users targeted scientific fertilizer and spraying guiding effect ${ }^{[2] .}$

\section{Basic geographic function application}

Features provided by the system to show the whole map and window shows the map with the map the location of the relationship, for the layer window display control, map coordinates with the movement of the mouse, the dynamic display referred to in the mouse position of longitude and latitude, as the map zoom in and out, dynamic display of the current map scale; The content also changes ${ }^{[3]}$.

(1) map display function

Users can in the layer control layer to select the required display of window, when the check box 
in the layer control window is selected, the layer's depiction of the detailed information will be displayed in the map display window. The layer can be separated by text, color or symbol size depending on the situation. As in figure 2, the "xianjie" layer is marked with different colors depending on the name of each place.

(2) map enlargement function

Click "enlarge" button and the cursor is magnifying glass. After that, when a mouse clicks on a map, the map will be displayed at the center magnification of a scale of scale. As the map enlarges, the number of layers that can be displayed will gradually increase, and the content of electronic map will be more and more abundant. In this way, by adjusting the amplification level, the overall global map and the local effect of the large scale map are achieved on the computer screen. To achieve the best display effect, the map is enlarged several times and no longer enlarged.

(3) map reduction function

Click the "shrink" button and the cursor is narrowed. Click the left mouse button on the map, and the map will be reduced by a scale of one size at that point. With the shrinking of the map, the number of layers and content of the objects are reduced correspondingly, and the local map is reduced to less than the full picture. If the map is in the middle of the window, the system automatically pulls the map back to the center of the window to display ${ }^{[4]}$.

(4) map roaming function

After map zoom, visible content is limited in the display window, then click on the "roaming" button, the cursor is hand, move the cursor to a location by pressing the left mouse button to drag on the screen, the map will be roaming in a row, in the direction of drag the map scale and the layer number remain the same. The purpose of roaming can also be achieved by operating in the thumbnail window.

(5) map thumbnail function

The thumbnail window displays the thumbnail of the electronic map according to the full figure, and the thumbnail has a rectangular box representing the current display in the map display window. Drag the rectangle box with the mouse, and when the rectangle is moved to the area where the user needs it, the map displays the contents of the corresponding position quickly in the map display window.

(6) map movement function

There are four arrowheads in the toolbox, and the directions of these four buttons are in the direction of the map movement. We can press a button to move the map to a certain distance.

(7) map measuring function

When we want to know from one place to another place or go through a few places have how far, you can select the measuring tool, as long as the progressive click go through, you can get the distance between them and the total distance ${ }^{[5]}$. Factor selection: in the feature selection function, the user can select elements by rectangle, circle, polygon and line. Before select the elements of the selected layer, and then select the elements by which way to choose, then in the map window, set the selection area, has been selected area contains elements will be selected.Property query: the user selects the layer to query the property first and then selects the property query button in the toolbox, which displays all the attribute information of the layer shown below. See figure 1

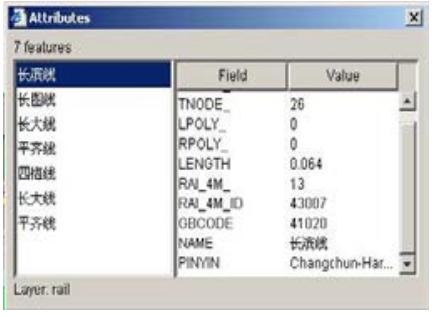

Fig1 Query property

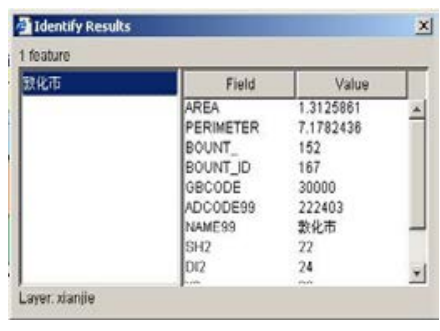

Fig 2 Query each other

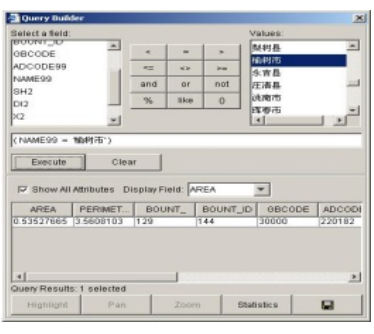

Fig 3 query tool

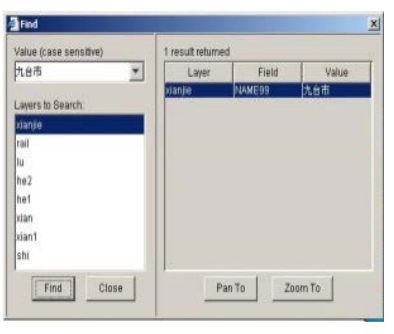

Fig 4 find tool

Map attribute peer review: through the elements of the query attribute (identify): the user first choice to query the layer, select the identify tool again, and then select the layers in the map 
window of the attribute of an element. As shown in figure 2 attribute query elements (query) : the user first choice to query the layer, select query tool again, will display window, as shown in the window screen input query conditions, select the execute button again, will satisfy all the condition attribute information, and will choose to meet the conditions of the layer in the map window elements ${ }^{[6]}$. See figure 3.

Attribute query elements (find) : the user to select a find tools, find in the query window, the first choice to query layer and attribute value of input query again, click the find button, in the results window will be displayed on the right information layer, fields, and the value of an attribute, below the window, there are two buttons (scaling to peace moved to). By choosing to shift to the button, it will be shifted to the selected elements; Zooming to the button will enlarge or shrink to the selected elements. See figure 4.

\section{Intelligent decision-making application}

The function realizes the fertilizing of the relevant plot according to the nutrient content and the target yield inference. After the form shown in figure 5, enter the target output, click the inference button, and the amount of fertilizer in the area will be given out. According to the amount of fertilizer in each plot given by the decision, the fertilization prescription map of the corresponding plot is plotted to provide fertilizer application for the fertilizer application. FIG. 6 is a recipe map for the fertilization of no.7 village no.13 village, bowen town, yuci city, 2009.

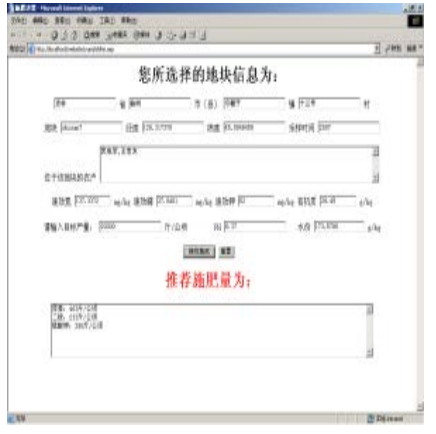

Fig5 decision fertilization;

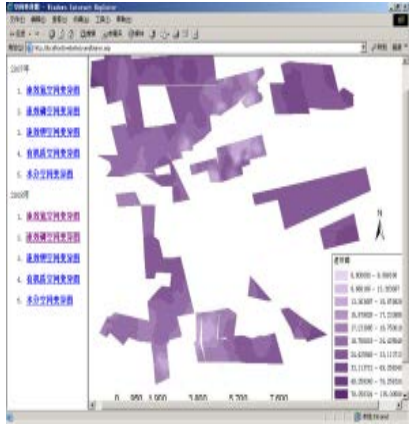

Fig 7 Variation map;

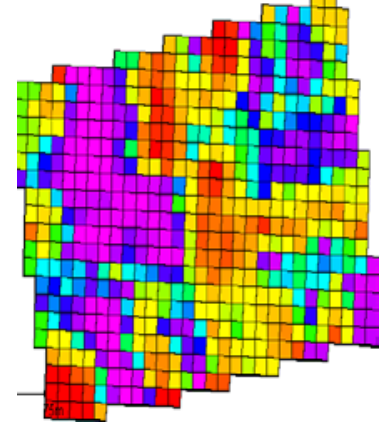

Fig 6 fertilizer prescription map;

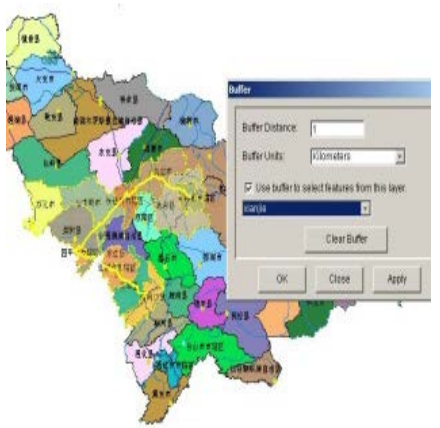

Fig 8 Buffer Analysis

\section{Spatial analysis application}

(1) spatial difference analysis

The spatial variation of the soil velocity nitrogen, instant phosphorus, instant potassium, organic matter, $\mathrm{PH}$ value, water content and yield was generated by spatial analysis of the nutrient and yield measured ${ }^{[7]}$. Click the variation diagram button to enter the page, select the year and some diagram to display the result. 


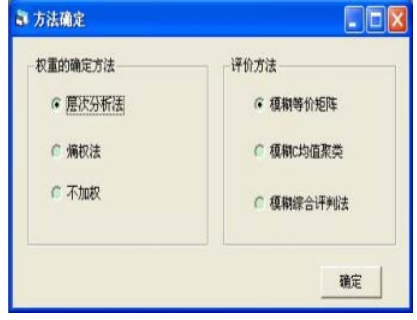

Fig 9 Methods choose;

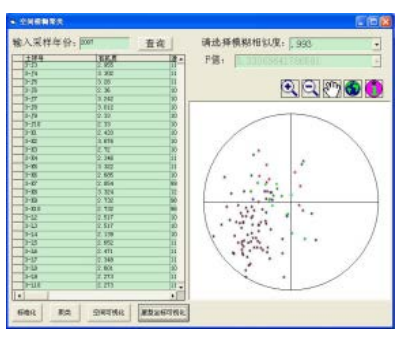

Fig 11 clustering visualization Based

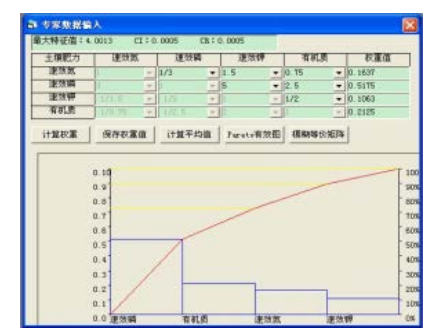

Fig 10 Weight Calculation;

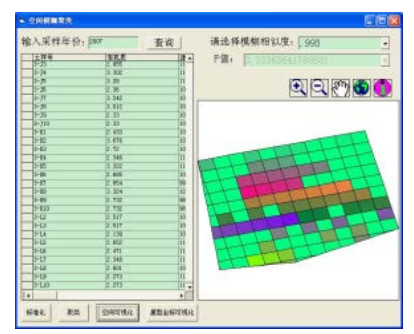

Fig 12 clustering visualization based on GIS

(2) buffer analysis

The user selects the layer and selects the previous element, then selects the buffer tool, sets the parameters (buffer distance and unit) and the layer of the buffer, and clicks the application or determines. The result is shown in figure 9.

\section{Application of visual weighted fuzzy clustering system}

The first step to the system is to select the method, as shown in figure 9. Main selected in this window the determination of weight coefficient method and evaluation method of the data, the following with analytic hierarchy process (ahp) and fuzzy clustering algorithm based on fuzzy equivalence matrix as an example, introduce the use of the system. When the selection is completed, click the ok button to enter the hierarchical analysis algorithm interface, add evaluation indexes on this page, and then enter the expert scoring evaluation window.

In this window, experts according to the importance of the two indexes, grade evaluation, this system according to the importance of the index contrast, built in 1/9, 1/8, 1/8, 1/6, 1/5, 1/4, 1/3, 1/2, $1,2,3,4,5,6,7,8,9$ options, such as options without proper human input). After the scoring, click the calculation weight button to calculate the weight and consistency test index of each indicator. Click the fuzzy equivalent matrix button to enter the space fuzzy cluster window ${ }^{[8]}$.

In the space fuzzy clustering window shown in figure 12, enter the data year to be evaluated, and the results are displayed in the data table. Click the standardized button to standardize the evaluation data; By clicking the clustering button, the normalized data can be calculated and the fuzzy similarity matrix is obtained. According to the fuzzy equivalence matrix, the fuzzy similarity is found and its value is added to the drop-down list box. , select the appropriate fuzzy similarity can be calculated as the classification of the situation, and according to the classification of calculate the probability and statistics in the distribution of the F F value, this value can reflect this kind of classification is significant; After the classification, the data mining visualization techniques can be used to visualize the classification results. FIG. 12 shows the visual results of [7] in star coordinates for the fuzzy similarity of 0.993 .

\section{Examples of application}

(1) the application of spatial weighted fuzzy clustering algorithm in evaluating the effect of variable fertilization

First of all, the analytic hierarchy process of 6.5 was used to obtain the weight of organic matter, instant nitrogen, instant phosphorus and potassium in the soil in the soil, respectively, 0.2105, $0.1579,0.5263$ and 0.1053 respectively. Then, using the weighted clustering algorithm, respectively 
for before variable fertilization (2005), continuous variable fertilization 2 years (2007) and continuous variable fertilization five years (2012) of soil organic matter, available nitrogen, available phosphorus and available potassium four nutrient data clustering analysis, the clustering results are shown in table 1 . In the table, the similarity between the data is represented, the class number is the classification number corresponding to the similarity, $\mathrm{F}$ - value is the value of $\mathrm{F}$ distribution calculated by probability statistics. The larger the $\mathrm{F}$ value, the greater the distance between classes and classes, the better the classification is.

In yushu city GongPeng town village 3 to 13 in 2005, 2008 and 2012, the soil organic matter, available nitrogen, available phosphorus and available potassium weighted fuzzy clustering results of four nutrient data comparison, learned that:

After continuous variable fertilization, the range of the values gradually decreased, and the similarity between the data gradually gathered, so the comprehensive difference in the number 3 was decreasing year by year.

When all the data were divided into one category, the value rose from 0.91 in 2005 to 0.928 in 2008, to 0.958 in 2012. After continuous variable fertilization, the comprehensive similarity of organic matter, instant nitrogen, instant phosphorus and potassium in the soil of the whole lot increased year by year.

When the time is, the $\mathrm{F}$ - value of three years is bigger, the classification is better. At this point, the number of categories in 2009 and 2012 was smaller than 2005, indicating that the distribution of soil nutrients was more concentrated than in 2005. And although the classification of the 2008 and 2012 are equal, but in 2008 the $\mathrm{F}$ value, reflects the gap between its classes and class than in 2012, in 2012 the soil nutrient data more close ${ }^{[9]}$. According to the spatial relationship of each grid, the results of clustering results are analyzed in GIS

Table 1 Clustering results table

\begin{tabular}{lcc|ccc|rrr}
\hline \multicolumn{3}{c|}{ 2005 year } & \multicolumn{3}{c|}{ 2008 year } & \multicolumn{3}{c}{ 2012 year } \\
\hline$\lambda$ & Class number & F- value & $\lambda$ & Class number & F- value & $\lambda$ & Class number & F- value \\
\hline 1 & 104 & 0.455 & 1 & 116 & 0.323 & 1 & 113 & 0.339 \\
0.999 & 92 & 0.895 & 0.999 & 104 & 0.716 & 0.999 & 100 & 0.652 \\
0.998 & 76 & 1.596 & 0.998 & 81 & 1.391 & 0.998 & 86 & 1.305 \\
0.997 & 62 & 2.596 & 0.997 & 62 & 2.764 & 0.997 & 71 & 2.304 \\
0.996 & 56 & 3.142 & 0.996 & 48 & 4.645 & 0.996 & 48 & 3.445 \\
0.995 & 49 & 4.151 & 0.995 & 42 & 5.739 & 0.995 & 40 & 4.594 \\
0.994 & 34 & 4.608 & 0.994 & 32 & 6.941 & 0.994 & 32 & 4.875 \\
0.993 & 31 & 5.064 & 0.993 & 28 & 8.053 & 0.993 & 28 & 4.898 \\
0.992 & 29 & 4.786 & 0.992 & 23 & 1.512 & 0.992 & 23 & 1.371 \\
0.991 & 25 & 5.300 & 0.991 & 21 & 1.424 & 0.991 & 21 & 1.469 \\
0.99 & 23 & 5.391 & 0.99 & 17 & 1.448 & 0.99 & 17 & 1.543 \\
0.989 & 22 & 5.207 & 0.989 & 15 & 1.327 & 0.989 & 15 & 1.084 \\
0.988 & 20 & 5.386 & 0.988 & 13 & 1.422 & 0.988 & 13 & 1.069 \\
0.987 & 17 & 5.265 & 0.987 & 12 & 1.372 & 0.987 & 9 & 1.121 \\
0.986 & 15 & 1.512 & 0.986 & 11 & 1.372 & 0.982 & 7 & 0.935 \\
0.985 & 13 & 1.748 & 0.985 & 10 & 1.323 & 0.981 & 6 & 0.920 \\
0.984 & 11 & 1.847 & 0.98 & 9 & 1.244 & 0.978 & 4 & 0.983 \\
0.982 & 10 & 1.907 & 0.978 & 8 & 1.237 & 0.977 & 3 & 0.885 \\
0.981 & 9 & 2.043 & 0.977 & 7 & 1.344 & 0.974 & 2 & 1.086 \\
0.98 & 7 & 1.957 & 0.974 & 6 & 1.454 & 0.958 & 1 & 1 \\
\hline
\end{tabular}

(2) the application of entropy weight method weighted space fuzzy clustering in the evaluation of land force

In the third chapter, the entropy weight method weighted spatial fuzzy clustering algorithm is verified by the benchmark data set, which proves that the algorithm can improve the precision of fuzzy clustering. Therefore, it is feasible to apply this algorithm to the earth force evaluation.

Selection of jilin province county agriculture administrative map, soil map, and test of $\mathrm{PH}$, organic matter, total $\mathrm{n}$, available $\mathrm{p}$, available $\mathrm{k}$, slowly put $\mathrm{k}$, effective state (copper, zinc, iron, manganese, boron, molybdenum) etc in the data as the basis for evaluation data set (data from jilin province county agriculture agricultural technology extending stations). First, the selected data is 
cleaned and the data set that meets the evaluation criteria is selected (table 2 is the partial data after cleaning).

Table 2 part of productivity evaluation data

\begin{tabular}{cccccccccccr}
\hline PH & CU & FE & HK & K & MN & N & P & YJZ & YLZ & ZN & production \\
\hline 6.6 & 1.25 & 8.72 & 838.2 & 146.0 & 22.33 & 0.095 & 15.4 & 2.12 & 19.92 & 1.35 & 507.5 \\
6.7 & 1.26 & 8.47 & 848.4 & 147.9 & 22.41 & 0.096 & 15.1 & 2.13 & 20.18 & 1.34 & 467.1 \\
6.7 & 1.39 & 4.70 & 844.8 & 161.3 & 22.05 & 0.102 & 13.0 & 2.18 & 21.60 & 1.35 & 544.0 \\
6.5 & 1.21 & 9.45 & 812.4 & 141.8 & 22.44 & 0.092 & 16.4 & 2.07 & 19.25 & 1.37 & 545.7 \\
6.7 & 1.27 & 9.71 & 861.6 & 149.2 & 21.88 & 0.098 & 14.2 & 2.15 & 20.71 & 1.33 & 523.9 \\
6.6 & 1.20 & 8.30 & 800.9 & 138.7 & 21.25 & 0.088 & 17.1 & 2.02 & 18.58 & 1.44 & 539.5 \\
6.6 & 1.30 & 8.50 & 821.7 & 144.8 & 22.30 & 0.093 & 15.9 & 2.11 & 19.50 & 1.39 & 537.2 \\
6.7 & 1.28 & 8.48 & 865.2 & 148.8 & 21.89 & 0.097 & 14.3 & 2.16 & 20.61 & 1.34 & 478.8 \\
6.7 & 1.30 & 0.10 & 862.7 & 152.9 & 21.90 & 0.099 & 13.8 & 2.17 & 20.96 & 1.35 & 478.5 \\
6.6 & 1.27 & 8.28 & 834.8 & 145.8 & 22.35 & 0.094 & 15.5 & 2.12 & 19.77 & 1.37 & 520.0 \\
6.6 & 1.28 & 8.34 & 829.8 & 145.8 & 22.33 & 0.094 & 15.6 & 2.12 & 19.66 & 1.39 & 512.6 \\
6.7 & 1.29 & 7.95 & 863.4 & 147.9 & 22.00 & 0.096 & 14.3 & 2.17 & 20.37 & 1.36 & 475.9 \\
6.7 & 1.37 & 1.50 & 855.2 & 157.8 & 22.00 & 0.100 & 13.2 & 2.19 & 21.24 & 1.37 & 505.6 \\
6.7 & 1.31 & 8.13 & 846.3 & 146.9 & 22.22 & 0.095 & 14.8 & 2.15 & 20.02 & 1.38 & 541.9 \\
6.8 & 1.32 & 8.71 & 863.4 & 151.6 & 21.85 & 0.098 & 14.0 & 2.19 & 20.62 & 1.39 & 480.4 \\
6.7 & 1.38 & 3.20 & 849.6 & 160.3 & 21.98 & 0.102 & 13.0 & 2.19 & 21.42 & 1.36 & 456.1 \\
6.5 & 1.34 & 8.43 & 803.1 & 145.4 & 22.08 & 0.092 & 16.4 & 2.10 & 19.03 & 1.45 & 506.6 \\
6.7 & 1.36 & 9.31 & 848.7 & 152.7 & 21.80 & 0.098 & 14.2 & 2.19 & 20.53 & 1.42 & 513.1 \\
\hline
\end{tabular}

Then, entropy weight method weighted spatial fuzzy clustering algorithm is used to evaluate the data set. Finally, using GIS to visualize the evaluation results, we can get the evaluation results of cultivated land force in the county of nongan county as shown in figure 13.

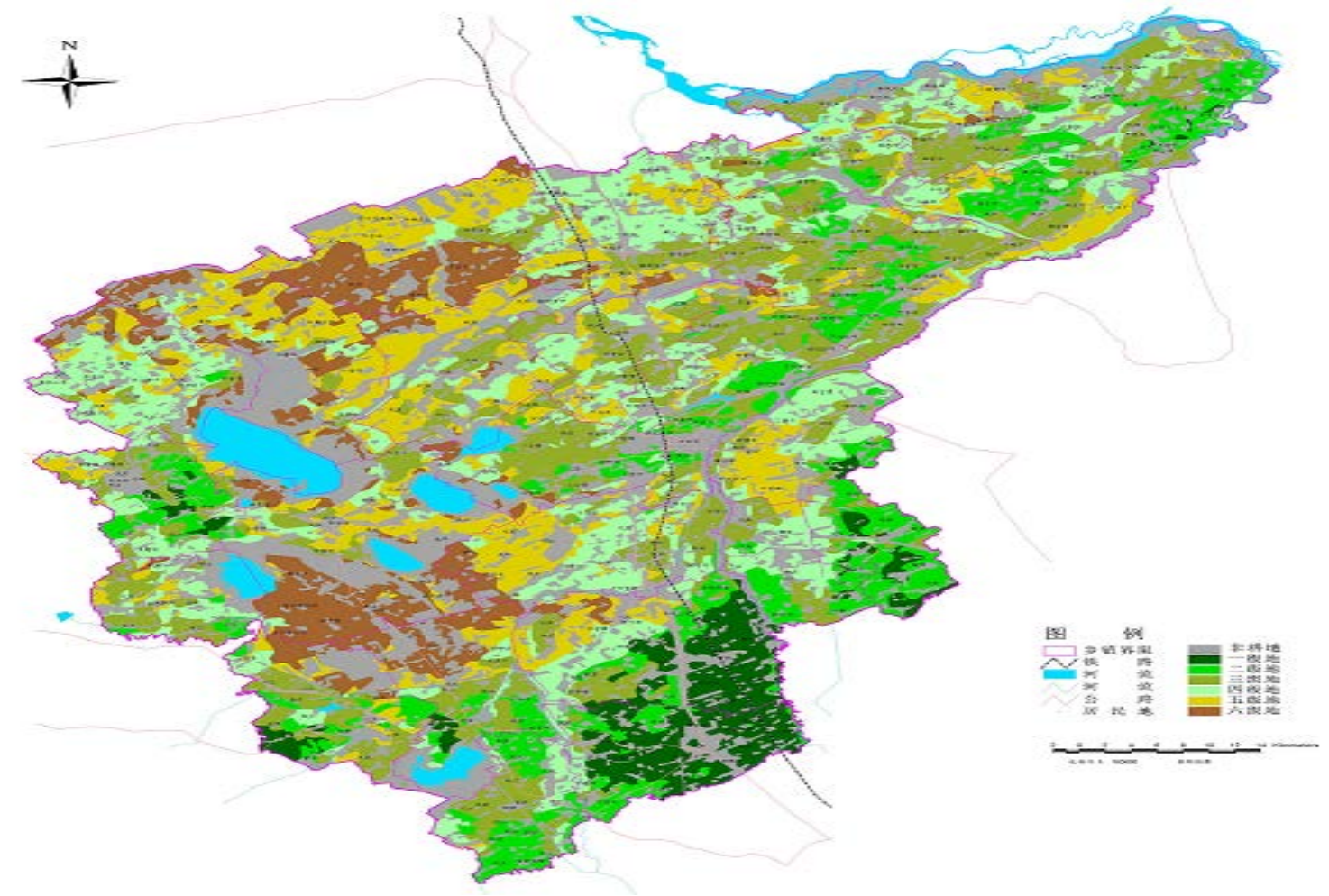

Fig 13 productivity evaluation result

Introduces the WebGIS system in this paper, spatial data mining and visualization of data mining method, the integrated application of WebGIS, expert system, decision support system and visual space fuzzy data mining technology, developed a "VSDM and WebGIS based on Internet + corn 
intelligent decision-making system" ${ }^{[10]}$. The application of web gis, expert system, decision support system and visual spatial fuzzy data mining is applied to the system implementation, so as to achieve the management, analysis and decision-making of spatial data. Combined with hierarchy analysis method, this paper proposes a space weighted fuzzy dynamic clustering algorithm, the classification results of application of the algorithm, the practical problems of classification more objective and accurate, algorithm, by introducing the F - distribution in the fully consider the various between and within class, on the basis of the difference of the optimal threshold, and use the best threshold value is the best classification; Based on entropy weight method in information theory, a new algorithm for FCM algorithm is proposed by using entropy weight method. The entropy weight method is the difference between the data itself and the actual distribution of data. The visualization of spatial data mining results in two ways can help us to analyze and make decisions on data. According to the nutrient content of the community, the fertilization decision was realized, and the fertilizer rate was obtained. Based on this, the application of fertilization recipe is generated to provide the data basis for the operation of the variable fertilizer machine.

Currently, the function of the theoretical research has been completed, the algorithm of each part is programming implementation, and as long as a little modification, the algorithm can be applied to other fields, has good portability. However, the weighted FCM algorithm has not been integrated into the system yet.

\section{References}

[1] Yao Yuxia, based on the research and application of the expert system for the diagnosis and treatment of cordyceps in jilin corn, [J] jilin agriculture, 2014 (10) 102-103.

[2] Chen Guifen,The research and application of the accurate fertilization system of maize [J], journal of jilin agricultural university, 2006:586-590.

[3] Wu Yuhua, ye ping. Research on the promotion model of "intelligent decision-making system for corn production management" [J]. Chinese agriculture bulletin. 2009, 25 (12) : 274-277.

[4] Zhangyun,Application of computer technology in maize production implemention precision agricultion[J]2014IEEE Workshaop on Advanced Research and Technology Industry Applications(WARTIA) OTTAWA CANADA， 2014(9)1161-1165.

[5] Li Ping, Wei chaofu et al. Evaluation of regional land development and sorting potential based on AHP and entropy weight method [J]. Journal of agricultural engineering, 2009, 25 (5) : 202-209.

[6] Yao yuxia, research and practice of corn yield prediction based on remote sensing technology, heilongjiang technical information, 2013(2)87-89.

[7] Yuxia-yao,The Research and Application of Remote Sensing Technology in MaizeYield Estimation of Jilin, [J]2014IEEE Workshaop on Advanced Research and Technology Industry Applications CANADA2014(9)1068-1071

[8] Wu Yuhua, ye ping. Analysis of restriction factors for the promotion of "intelligent decision-making system for corn production management" [J]. China agriculture bulletin. 2009, 25 (10) : 258-263.

[9] li Yajun, the diagnostic system of corn disease, [J] agricultural disaster research, 2012 (5) 129-130.

[10] Yao Yuxia, relying on intelligent management technology to improve maize production capacity, [J] jilin agriculture, 2016. (12) 63-64. 